
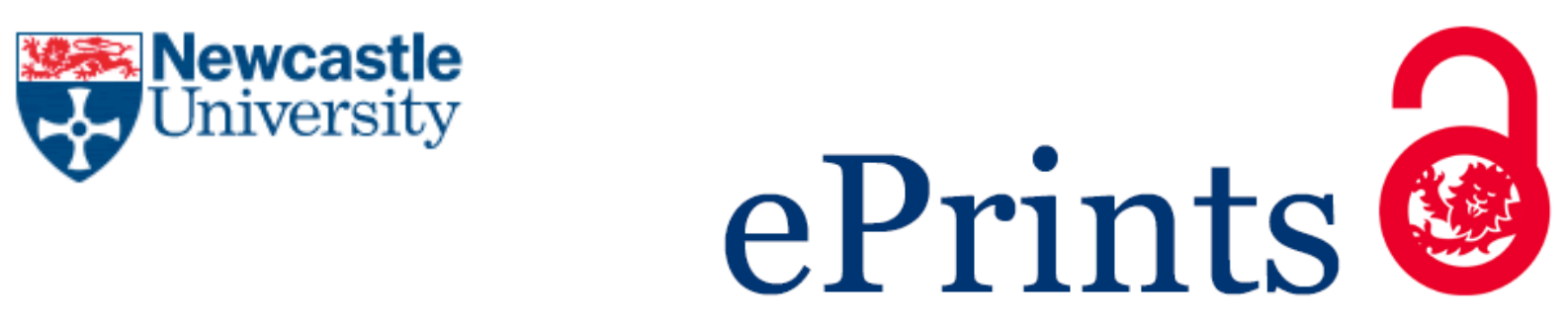

\author{
Mullan F, Austis RS, Parkinson CR, Bartlett DW. \\ An in-situ pilot study to investigate the native clinical resistance of \\ enamel to erosion. \\ Journal of Dentistry 2018 \\ DOI: https://doi.org/10.1016/j.jdent.2018.01.005
}

\title{
Copyright:
}

(C) 2018. This manuscript version is made available under the CC-BY-NC-ND 4.0 license

DOI link to article:

https://doi.org/10.1016/j.jdent.2018.01.005

Date deposited:

$19 / 01 / 2018$

Embargo release date:

12 January 2019

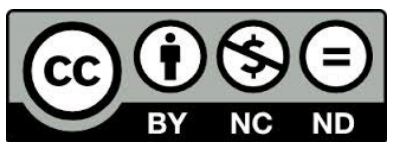

This work is licensed under a

Creative Commons Attribution-NonCommercial-NoDerivatives 4.0 International licence 


\section{An in-situ pilot study to investigate the native clinical resistance of enamel to erosion}

Francesca Mullan*, Rupert. S. Austin, Charles. R. Parkinson and David. W. Bartlett

\section{Introduction}

Human enamel has a complex hierarchal microstructure, constituting $96 \%$ mineral, $3 \%$ water and $1 \%$ organic protein [1]. The mineral content is a hydroxyapatite, sometimes referred to as a calcium deficient hydroxyapatite (HA) due to the constant dynamic flux of metal ions occurring with the oral environment $[2,3]$. Prior to tooth eruption, secretory ameloblasts form the fundamental microstructural unit of enamel. The enamel rod or prism, runs from the edge of the inner dentine to the outer enamel surface, having only 3-7 $\mu \mathrm{m}$ diameter whilst up to 4 $\mathrm{mm}$ length, with each prism interlocked by an organic protein complex $[1,4]$. The orientation and alignment of the prisms as they course within the enamel are so variable that it is very difficult to trace any individual prism's path through the tooth [5-7]. However, some important micro-structural patterns are known, such that each prism interlocks in a honeycomb or fish-scale like pattern and travels in a sinusoidal pattern towards the surface to decussate perpendicular to the occlusal surface whereas on the axial surfaces of the tooth the prisms are angulated around $60^{\circ}$ the surface [8]. Ultra-structurally within the prism, the orientation of crystallites are not uniform $[9,10]$ and further structural considerations include Retzius lines and prism cross striations which are linked to the formation of the enamel [7].

All these variations affect the biomechanical performance of the enamel as it undergoes wear whether from chemical (acid erosion) or mechanical (attrition and abrasion) challenges. In 
vitro erosive tooth wear models normally employ polishing of enamel to remove the outer layer to reduce structural variations, and thus facilitate measurement [11]. However, this means the outer layer, which contains areas of aprismatic and prismatic enamel which is less susceptible to erosion [12-16], has been removed and therefore the erosion model maybe less clinically relevant.

However, the measurement of enamel in its naturally curved state has perceived difficulties. Contact and optical systems map the surface either using a stylus or a light source combined with software containing specific algorithms [17]. However, for both types of system the overall shape and type of surface can affect the measurement capability. Specifically, accuracy which is the closeness in agreement to a measured value or its 'true' value, whereas, resolution is smallest detectable measurement that a device can record and finally precision is the closeness in agreement to a series of measurements, and expressed as SD. All these factors differ for flat and curved surfaces $[18,19]$. Hewlett et al $1992[20]$ demonstrated by measuring a sphere gauge, using a contact profilometer, that accuracy and precision decreased over sloped areas. The light source of an optical system distorts and elongates over slopes reducing accuracy and precision [21]. It has previously been identified that profilometric step height measurements of natural enamel resulted in a precision (SD) of 3.9 $\mu \mathrm{m}$ compared with $2.2 \mu \mathrm{m}$ for polished enamel [22]. Surface roughness measurements have been advocated and increasingly used for the quantification of early erosive tooth wear, but with limited success in natural enamel samples $[15,23,24]$. In a study investigating the microstructure of dental hard tissues, Ranjitkar et al 2016 noted that measuring sloped regions resulted in increased drop out from the laser [25]. These developments have significance when trying to establish appropriate laboratory protocols to effectively assess 
the effects of enamel erosion. Whilst studies suggest that natural enamel is more resistant to roughness change, to fully interpret this type of data the measurement error for both enamel substrates (natural and polished) must also be investigated, which so far has not been done.

The aims of this study were to determine the precision of surface roughness measurements of naturally curved (natural) enamel and polished flat enamel and to compare the surface effects of a dietary erosive challenge on the 3D roughness of these two types on enamel substrates.

\section{Methods}

Forty-two enamel sections ( $5 \mathrm{~mm} \times 5 \mathrm{~mm} \times 3 \mathrm{~mm}$ ) were prepared from the mid-buccal aspect of sound human molars donated under ethical agreement (REC: 12/LO/1836) and embedded in bisacryl composite material (Protemp4 ${ }^{\mathrm{TM}} 3 \mathrm{M}$ ESPE, Germany) prior to random allocation to either natural or polishing. Twenty-one enamel samples were partially embedded with their maximum buccal convexity positioned centrally leaving their outer surface accessible to ultrasonication and alcohol cleaning, thus resulting in $3 \mathrm{~mm} \times 2 \mathrm{~mm}$ of natural exposed enamel, as shown in Error! Reference source not found.. The remaining twenty-one enamel samples were fully submerged and polished to a flatness tolerance of $0.4 \mu \mathrm{m}$ using a series of silica carbide grits and a water cooled rotating polishing machine (Struers LaboPol-30, Struers, Copenhagen, Denmark) and ultrasonicated on completion of polishing regime following previously published protocols [11].

A 655 nm Confocal Laser Displacement sensor (LT-9010M, Keyence Corporation, Japan) and motion controlled profilometry stage (Xyris 2000, Taicaan, UK) were used throughout the study, in combination with surface metrology software (MountainsMap ${ }^{\circledR}$ v7.3, DigitalSurf, 
France) to measure 3D Sa surface roughness following ISO texture measurement standards [26]. The profilometer had a spot size of $2 \mu \mathrm{m}$ and vertical resolution of $100 \mathrm{~nm}$, with a $4 \mu \mathrm{m}$ lateral scanning interval. An image analysis workflow was designed to apply a $25 \mu \mathrm{m}$ robust Gaussian filter and extract 3D $(\mathrm{Sa})$ roughness values $(\mu \mathrm{m})$ centred around the centre of the polished enamel surface and the zenith (maximum bulbosity) of the natural enamel sample surface as shown in Figure 1, following previously published protocols [27].

The precision of 3D surface texture measurement was determined by performing repeated scans following good practice measurement guidelines [28]. Five measurement areas (each $0.04 \mathrm{~mm}^{2}$ ) were selected from the centre of each sample using an image acquisition macro written within the motion control software $\left(\right.$ Stages $^{\mathrm{TM}}$, TaiCaan Technologies, Southampton, UK) to ensure consecutive repeated scanning of each area 30 times, thus ensuring there were no changes to conditions. Following image acquisition, the mean (SD) Sa surface roughness (MountainsMap ${ }^{\circledR}$ v7.3, DigitalSurf, France) of the 30 scans of each area measured and the precision was expressed by calculating the variability of measurement (i.e. SD in nm) [29].

To simulate dietary wear an erosion model was designed to investigate surface roughness changes following in vitro exposure to dietary acid, based upon previously published protocols $[14,27]$. A 3-cycle erosion regime was investigated using a total of 40 enamel samples (20 polished and 20 natural). Enamel samples were fully immersed in $100 \mathrm{~mL}$ of a commercial orange juice with pH 3.2 and titratable acidity $41.3 \mathrm{mmol} \mathrm{OH} / \mathrm{L}$ (Sainsbury's basic orange juice drink, Sainsbury's, London, UK) for 15 minutes per cycle under constant agitation at 62 rpm using an orbital shaker (Stuart Scientific, Mini Orbital Shaker S05, Bibby), thus giving a total erosion time of 45 minutes. A single operator located and analysed five scan areas per sample, 
at baseline and after erosion, in order to calculate the mean (SD) Sa surface roughness change $(\mu \mathrm{m})$, as shown in Figure 1.

To qualitatively characterise the enamel surface changes, representative confocal images were acquired from polished and natural samples using tandem scanning microscopy in white light reflection mode (Noran Instruments, Middleton, WI, USA) in conjunction with a $\times 20$ objective lens (M-Plan SLWD Brightfield $\times 20 / 0.35$ NA) and an automatic z-stage piezo electric controller (E-662.SR LVPZT Piezo Amplifier/Position Servo Controller, Physik Instrumente, Germany). For each sample an $85 \mu \mathrm{m} \mathrm{Z} \mathrm{stack} \mathrm{at} 0.5 \mu \mathrm{m}$ intervals was acquired using proprietary image acquisition software (Micromanager v1.4.22, Open Imaging; Inc. San Francisco, CA, USA) in order to capture information from the surface and immediate subsurface $(<85 \mu \mathrm{m})$ enamel. The resulting stack of $2 \mathrm{D}$ images was processed by a stack analyser programme (ImageJ, US National Institutes of Health, Bethesda, MD) to produce an optimised $533 \mu \mathrm{m} \times 533 \mu \mathrm{m}$ pseudo-topography image for qualitative texture analysis.

\subsection{Statistical analysis}

Precision was expressed by calculating the variability (SD) of the 30 repeated measurements $(\mathrm{nm})$ for each of the 5 analysis areas. 3D roughness data were exported from the surface analysis software to spreadsheet software (Microsoft Excel 2010) and the mean Sa roughness of the five measurement areas was calculated $(\mu \mathrm{m})$ per sample for statistical analyses. Histogram plots were used to data were normally distributed, data sets which were not normally distributed were log transformed if positively skewed. F tests were used to compare the variability (standard deviation) and $\mathrm{P}<0.05$ considered statistically significant.

For the analysis of 3D surface roughness during erosion, the mean Sa from the five measurement areas were calculated per sample prior to calculation of the group change in Sa 
roughness before vs. after erosion. The group roughness change data were normally distributed therefore Independent T Tests were used to compare Sa roughness changes before vs. after erosion times and $\mathrm{P}<0.05$ considered statistically significant.

\section{Results}

Table 1 shows the precision of 3D enamel surface (Sa) roughness measurements. The data shows optimal precision at the central measurement area (Area 3) for both the polished enamel $(1 \mathrm{~nm})$ and natural enamel $(7 \mathrm{~nm})$ and worst precision of $5 \mathrm{~nm}$ for the natural enamel and $23 \mathrm{~nm}$ for the polished enamel. Therefore, for polished enamel there were statistical differences between the precision of measurement for the top left and bottom right measurement areas (i.e. 1 vs 5) $(P<0.001)$, top right and bottom left measurement areas (i.e. 2 vs 4$)(P<0.05)$ and the central and bottom left measurement areas (i.e. 3 vs 4$)(P<0.001)$. Equally for the natural enamel, there were statistical differences between the precision of measurement for the central; top right; bottom left and bottom right measurement areas (i.e. 1 vs 3,4 and 5) $(P<0.001)$ and the central; top right and bottom right measurement areas (i.e. 2 vs 3,5$)(P<0.001)$

The results of the surface texture changes after the erosion are shown in Table 2 . The polished enamel samples displayed a statistically significant increase of $48 \%$ in roughness after erosion resulting in a mean (SD) roughness change of $0.10(0.07) \mu \mathrm{m}$ after 45 minutes immersion in orange juice $(\mathrm{P}<0.05)$. In contrast, natural enamel displayed almost the opposite trend with a statistically significant $45 \%$ decrease in roughness and thus became significantly smoother, resulting in a mean $(S D)$ roughness change of $-0.32(0.42) \mu \mathrm{m}$ after 45 minutes immersion in orange juice $(P<0.05)$. 
Figure 2 shows the confocal images acquired from the $85 \mu \mathrm{m}$ of surface/subsurface enamel surfaces which revealed substantially different baseline surface features prior to erosion. The polished enamel surface before erosion was essentially featureless with no discernible microstructural histological features evident, whereas the natural enamel surface was highly textured. The natural enamel surface displayed smaller scale surface features which took the form of a round honeycomb like lattice, superimposed on longer range features which had the appearance of undulating hills and dales, which were in turn superimposed on an irregularly curved profile of the natural enamel surface. However, the appearance of the two surfaces after erosion revealed greater similarities, with both revealing a predominance of very smaller features across the entirety of the enamel surface which was appearing to obliterate the longer range features seen in the natural enamel surface prior to erosion.

\section{Discussion}

This study identified that the optical measurement system could reliably identify Sa roughness changes as small as $5 \mathrm{~nm}$ for polished enamel and $23 \mathrm{~nm}$ for natural enamel. Whilst there were differences in the precision of measurement across the five measurement areas, these differences were minimal of $4 \mathrm{~nm}$ for polished and $16 \mathrm{~nm}$ for natural enamel therefore suggesting that the level of precision is within the limits of detection required for reliable measurement of 3D surface texture changes of enamel undergoing erosion $[18,20,27]$.

There has recently been increased interest in investigating surface changes of natural enamel surfaces which are a more clinically relevant substrate [14,15,31,32]. However, difficulties have been identified specific to optical devices, whereby measurement drop out occurs over curvatures with push the angular tolerance of the device [25]. Previously, we conducted a 
study comparing the Sa roughness over different locations of natural and polished enamel samples, identifying that measurements from the centre of a natural enamel sample could be considered representative of the overall sample [27]. This area could also be described as 'zenith' or apex of the curvature which is attributed to area 3 of measurement in this study. This region had the highest level of precision for natural enamel. Therefore, this would reaffirm that to increase precision of measurement and allow genuine interpretation of surfaces changes of natural enamel, measurements should be taken from this 'zenith'. Interestingly there were statistically significant differences between the five areas for both natural and polished enamel, suggestive of the involvement of some element of biological variation in the surface enamel.

The level of roughness change detected for both polished enamel and natural enamel were within the capabilities of the device. By determining the equipment was capable of truly identifying changes at this level one can be confident that this statistical inference is indeed a true negative. Baseline values for natural enamel indicate a complex textured surface, which is supported by visual evidence from the confocal images, thereby the effect of roughness change value is partly determined by the baseline values and the variation within the sample micro-structure. Natural enamel became significantly smoother after erosion whilst polished enamel became rougher, following recent trends $[15,24,27,33]$. These differences in behaviour between the two surfaces cannot be attributed to the differences in measurement capabilities and remain true representations. Therefore, they suggest natural enamel becoming smoother following acid exposure is a true quantification of the surface textural changes. Following erosion, the micro-histological textural features of natural enamel such as perikymata were reduced, the overall surface flattened and an increased number of enamel 
prisms exposed. This suggests that acid erosion of human enamel has the effect of eliminating longer wavelength features which dominate the natural enamel surface [34], however it is not yet known to what extent this may be used to prevent progression of erosion. Natural enamel has been described as being more resistant to erosion compared to polished enamel and certainly the effects of erosion are less pronounced $[12,13,15,16,33]$. This study supports this previous work and has identified that natural or polished enamel surfaces exhibit contrasting responses to a similar erosive challenges, thus suggesting that preserving the acidresistant outer layer of enamel may be essential for clinical management for erosive wear [35].

\section{Conclusion}

This study demonstrates a method for precise surface texture measurement of both polished and natural enamel. This opens up further possibilities for characterising the initial effects of acid erosion in natural enamel in order to develop optimal effective prevention methods prior to irreversible structural enamel loss occurs. Future studies should investigate the response of natural enamel to erosive and protective factors in order to provide a clinically representative model for greater understanding of the erosive process.

\section{Conflict of interest statement}

The authors declare that they have no conflict of interest.

\section{Acknowledgements}

Funding: This work was supported by GlaxoSmithKline 


\section{References}

[1] Cuy JLL, Mann ABB, Livi KJJ, Teaford MFF, Weihs TPP. Nanoindentation mapping of the mechanical properties of human molar tooth enamel. Arch Oral Biol 2002;47:281-91. doi:10.1016/S0003-9969(02)00006-7.

[2] Featherstone JDB, Lussi A. Understanding the chemistry of dental erosion. Monogr Oral Sci 2006;20:66-76. doi:10.1159/000093351.

[3] West NX, Joiner A. Enamel mineral loss. J Dent 2014;42 Suppl 1:S2-11. doi:10.1016/S0300-5712(14)50002-4.

[4] Ten Cate J.M, Larsen MJ, Pearce EIF, Fejerskov O. Chemical interactions between the tooth and oral fluids. In: Fejerskov O, Kidd EAM, editors. Dent. Caries. Dis. its Clin. Manag. 2nd ed., Blackwell Publishing Ltd; 2008, p. 209-31.

[5] Hirota F. Prism arrangement in human cusp enamel deduced by X-ray diffraction. Arch Oral Biol 1982;27:931-7. doi:10.1016/0003-9969(82)90099-1.

[6] Raue L, Gersdorff N, Rödiger M, Klein H. New insights in prism orientation within human enamel. Arch Oral Biol 2012;57:271-6. doi:10.1016/j.archoralbio.2011.08.015.

[7] Li C, Risnes S. SEM observations of Retzius lines and prism cross-striations in human dental enamel after different acid etching regimes. Arch Oral Biol 2004;49:45-52. doi:10.1016/S0003-9969(03)00195-X.

[8] Whittaker DK. Structural variations in the surface zone of human tooth enamel observed by scanning electron microscopy. Arch Oral Biol 1982;27:383-92. doi:10.1016/0003-9969(82)90147-9. 
[9] Al-Jawad M, Steuwer A, Kilcoyne SH, Shore RC, Cywinski R, Wood DJ. 2D mapping of texture and lattice parameters of dental enamel. Biomaterials 2007;28:2908-14. doi:10.1016/j.biomaterials.2007.02.019.

[10] Simmons LM, Al-Jawad M, Kilcoyne SH, Wood DJ. Distribution of enamel crystallite orientation through an entire tooth crown studied using synchrotron X-ray diffraction. Eur J Oral Sci 2011;119:19-24. doi:10.1111/j.1600-0722.2011.00909.x.

[11] Mistry M, Zhu S, Moazzez R, Donaldson N, Bartlett DW. Effect of model variables on in vitro erosion. Caries Res 2015;49:508-14. doi:10.1159/000438725.

[12] Meurman JH, Frank RM. Progression and surface ultrastructure of in vitro caused erosive lesions in human and bovine enamel. Caries Res 1991;25:81-7.

[13] Ganss C, Klimek J, Schwarz N. A comparative profilometric in vitro study of the susceptibility of polished and natural human enamel and dentine surfaces to erosive demineralization. Arch Oral Biol 2000;45:897-902. doi:10.1016/S00039969(00)00041-8.

[14] Mullan F, Bartlett D, Austin RS. Measurement uncertainty associated with chromatic confocal profilometry for 3D surface texture characterization of natural human enamel. Dent Mater 2017;33:e273-81. doi:10.1016/j.dental.2017.04.004.

[15] Hara ATT, Livengood SV V, Lippert F, Eckert GJJ, Ungar PSS. Dental Surface Texture Characterization Based on Erosive Tooth Wear Processes. J Dent Res 2016;95:537-42. doi:10.1177/0022034516629941.

[16] Lin WT, Kitasako Y, Nakashima S, Tagami J. A comparative study of the susceptibility of 
cut and uncut enamel to erosive demineralization. Dent Mater J 2017;36:48-53. doi:10.4012/dmj.2016-239.

[17] Field J, Waterhouse P, German M. Quantifying and qualifying surface changes on dental hard tissues in vitro. J Dent 2010;38:182-90. doi:10.1016/j.jdent.2010.01.002.

[18] Durakbasa MN, Osanna PH, Demircioglu P. The factors affecting surface roughness measurements of the machined flat and spherical surface structures - The geometry and the precision of the surface. Measurement 2011;44:1986-99. doi:10.1016/j.measurement.2011.08.020.

[19] Giusca CL, Leach RK. Calibration of the metrological characteristics of Imaging Confocal Microscopes (ICMs) 2013.

[20] Hewlett ER, Orro ME, Clark GT. Accuracy testing of three-dimensional digitizing systems. Dent Mater 1992;8:49-53. doi:10.1016/0109-5641(92)90053-F.

[21] Jovanovski V, Lynch E. Analysis of the morphology of oral structures from 3-D coordinate data. Monogroaphs Oral Sci 2000;17. doi:10.1159/000061641.

[22] Schlueter N, Ganss C, De Sanctis S, Klimek J. Evaluation of a profilometrical method for monitoring erosive tooth wear. Eur J Oral Sci 2005;113:505-11. doi:10.1111/j.16000722.2005.00253.x.

[23] Joshi M, Joshi N, Kathariya R, Angadi P, Raikar S. Techniques to Evaluate Dental Erosion: A Systematic Review of Literature. J Clin Diagn Res 2016;10:ZE01-ZE07. doi:10.7860/JCDR/2016/17996.8634.

[24] Arnold WH, Haddad B, Schaper K, Hagemann K, Lippold C, Danesh G. Enamel surface 
alterations after repeated conditioning with $\mathrm{HCl}$. Head Face Med 2015;11:32. doi:10.1186/s13005-015-0089-2.

[25] Ranjitkar S, Turan A, Mann A, Gully G., Marsman M, Edwards S, et al. Surface-Sensitive Microwear Texture Analysis of Attrition and Erosion. J Dent Res 2016:1-8.

[26] Blunt L (Liam), Jiang X. ISO 25178-2:2012(en) Geometrical product specifications (GPS) - Surface texture: Areal - Part 2: Terms, definitions and surface texture parameters, Kogan Page Science; 2012, p. 355.

[27] Mullan F, Austin RS, Parkinson CR, Hasan A, Bartlett DW. Measurement of surface roughness changes of unpolished and polished enamel following erosion. PLoS One 2017;12:e0182406. doi:10.1371/journal.pone.0182406.

[28] Bell S. Good Practice Guide No. 11 The Beginner's Guide to Uncertainty of Measurement n.d.

[29] Leach R. Fundamental Principles of Engineering Nanometrology. Elsevier Science; 2014.

[30] Rasband WS, S. W. ImageJ: Image processing and analysis in Java. Astrophys Source Code Libr Rec ascl1206013 2012.

[31] Borrero-Lopez O, Pajares A, Constantino PJ, Lawn BR. Mechanics of microwear traces in tooth enamel. Acta Biomater 2015;14:146-53. doi:10.1016/j.actbio.2014.11.047.

[32] Somasundaram G, Ribnick E, Sivalingam R, Eid A, Meyer T, Golnari G, et al. Detecting tooth wear using intra-oral 3d scans. WO2016003787 A1, 2015.

[33] Mullan F, Bartlett D, Austin RSRS. Measurement uncertainty associated with chromatic 
confocal profilometry for 3D surface texture characterization of natural human enamel. Dent Mater 2017;33:e273-81. doi:10.1016/j.dental.2017.04.004.

[34] Nanci A, Ten Cate AR (Arnold R. Ten Cate's oral histology : development, structure, and function. Mosby; 2008.

[35] Loomans B, Opdam N, Attin T, Bartlett D, Edelhoff D, Frankenberger R, et al. Severe Tooth Wear: European Consensus Statement on Management Guidelines. J Adhes Dent 2017;19:111-9. doi:10.3290/j.jad.a38102. 